\title{
Influence of home-based telemonitored Nordic walking training on autonomic nervous system balance in heart failure patients
}

\author{
Ewa Piotrowicz ${ }^{1}$, Teodor Buchner ${ }^{2}$, Walerian Piotrowski ${ }^{3}$, Ryszard Piotrowicz ${ }^{4}$
}

\begin{abstract}
${ }^{1}$ Telecardiology Centre, Institute of Cardiology, Warsaw, Poland ${ }^{2}$ Faculty of Physics, Warsaw University of Technology, Warsaw, Poland ${ }^{3}$ Statistics Laboratory, Institute of Cardiology, Warsaw, Poland

${ }^{4}$ Department of Cardiac Rehabilitation and Non-invasive Electrocardiology, Institute of Cardiology, Warsaw, Poland
\end{abstract}

Submitted: 14 September 2014

Accepted: 16 September 2014

Arch Med Sci 2015; 11, 6: 1205-1212

DOI: $10.5114 /$ aoms.2015.56346

Copyright @ 2015 Termedia \& Banach

\section{Abstract}

Introduction: Rehabilitation positively affects the modulation of the autonomic nervous system (ANS). There are no papers evaluating the influence of Nordic walking training (NW) on ANS activity among chronic heart failure (CHF) patients. The aim of study was to assess the influence of NW on ANS activity measured by heart rate variability (HRV) and heart rate turbulence (HRT) in CHF patients and its correlation with physical capacity improvement measured by peak oxygen consumption (peak $\mathrm{VO}_{2}[\mathrm{ml} / \mathrm{kg} / \mathrm{min}]$ ) in the cardiopulmonary exercise treadmill test (CPET).

Material and methods: The study group comprised $111 \mathrm{CHF}$ patients (NYHA class II-III; ejection fraction $(\mathrm{EF}) \leq 40 \%)$. Patients were randomized $(2: 1)$ to 8-week NW (five times weekly) at $40-70 \%$ of maximal heart rate (training group - TG) $(n=77)$, or to a control group (CG) $(n=34)$. The effectiveness of NW was assessed by changes (delta $(\Delta)$ ) in peak $\mathrm{VO}_{2}, \mathrm{HRV}$ and HRT as a result of comparing these parameters from the beginning and the end of the programme.

Results: Eventually, 36 TG patients and 15 CG patients were eligible for HRV and HRT analysis. In the TG low/high frequency ratio (LF/HF) decreased $(1.9 \pm 1.11$ vs. $1.7 \pm 0.63, p=0.0001)$ and peak VO increased $(16.98 \pm 4.02$ vs. $19.70 \pm 4.36 \mathrm{ml} / \mathrm{kg} / \mathrm{min}, p<0.0001)$. Favourable results in CG were not observed. The differences between TG and CG were significant: $\Delta$ peak $\mathrm{VO}_{2}$ $(p=0.0081) ; \Delta \mathrm{LF} / \mathrm{HF}(p=0.0038)$. An inverse correlation was found between the decrease in $\Delta \mathrm{LF} / \mathrm{HF}$ and the increase in $\Delta$ peak $\mathrm{VO}_{2}(R=-0.3830$, $p=0.0211$ ) only in the TG. Heart rate variability did not change significantly in either group.

Conclusions: Nordic walking positively affects the parasympathetic-sympathetic balance in CHF patients, which correlates with the improvement in $\Delta$ peak $\mathrm{VO}_{2}$. No significant influence of NW on HRT was observed.

Key words: exercise training, autonomic nervous system, heart rate variability, heart failure.

\section{Introduction}

Deranged autonomic nervous system (ANS) balance constitutes one of the numerous elements of the complex pathophysiological chronic heart failure (CHF) [1-4]. Most published studies indicate that well-planned

\author{
Corresponding author: \\ Dr. Ewa Piotrowicz \\ Telecardiology Centre \\ Institute of Cardiology \\ 42 Alpejska St \\ 04-628 Warsaw, Poland \\ Phone: +48223434664 \\ E-mail: epiotrowicz@ikard.pl
}


exercise training, apart from improving the physical capacity, is also capable of positively affecting the modulation of the majority of parameters (although not necessarily the same ones) which reflect the ANS activity in CHF patients [5-7]. This results from the fact that different exercise training modalities have been applied in the existing studies. There are no papers evaluating the influence of telerehabilitation based on Nordic walking training on ANS activity in CHF patients. It needs to be stressed that during the Nordic walking training, patients use the majority of their muscles and obtain fitness building stimulation not present in cycling or simple walking training [8]. Moreover, during this type of training patients also added a small resistance training component in the form of "grip and release" poles [8]. Additionally, breathing and light stretching exercises in between Nordic walking sessions can be performed. The non-invasive assessment of indices reflecting ANS activity on the surface electrocardiogram (ECG) allow one to evaluate the efficacy of Nordic walking training in terms of sympathetic and parasympathetic balance [9]. Therefore, the aim of this study was to assess the influence of Nordic walking training on ANS activity measured by heart rate variability (HRV) and heart rate turbulence (HRT) in CHF patients and its correlation with physical capacity improvement measured by peak oxygen consumption (peak $\mathrm{VO}_{2}$ ) evaluated in the cardiopulmonary exercise treadmill test (CPET).

\section{Material and methods}

The present study formed part of a single-centre, prospective, parallel-group, randomized $(2: 1)$, controlled trial of home-based telemonitored Nordic walking training (training group (TG)) vs. usual care (control group (CG)) in CHF patients [10] who also met the criteria for HRV and HRT evaluation. For this study, we recruited consecutive patients hospitalized between December 2009 and June 2012 in the Department of Cardiac Rehabilitation, Institute of Cardiology, Warsaw, Poland for inclusion in the cardiac rehabilitation. The study was approved by the local Ethics Committee. Each patient gave written informed consent.

We included: patients of either sex with any aetiology of left ventricular systolic CHF as defined in the European Society of Cardiology (ESC) guidelines [1] diagnosed at least for 3 months; with a left ventricular ejection fraction (LVEF) $<40 \%$ on echocardiography; classified by the New York Heart Association (NYHA) as class II or III; who were clinically stable and receiving an optimal and stable medication regimen for at least 4 weeks before enrolment; who had no contraindications to exercise training according to current recommendation for CHF patients [11].
The exclusion criteria were: a history of an acute coronary syndrome within the last month, coronary artery bypass grafting (CABG) within the last 2 months, or initiation of cardiac resynchronization therapy within the last 6 months, or implantation of a pacemaker and/or implantable cardioverter-defibrillator (ICD) within the last 6 weeks; symptomatic and/or exercise-induced cardiac arrhythmia or conduction disturbances; valvular or congenital heart disease requiring surgical treatment; hypertrophic cardiomyopathy; severe pulmonary hypertension or other severe pulmonary disease; uncontrolled hypertension; anaemia; acute and/or decompensated non-cardiac disease; physical disability related to severe musculoskeletal or neurological problems; patient refusal to participate.

Autonomic nervous system activity assessment was conducted in the eligible patients who were in sinus rhythm. The exclusion criteria were: atrial fibrillation or flutter, atrial and/or ventricular pacing.

The qualified patients in both groups underwent the following assessments at entry and after completing the 8-week programme: clinical examination, two-dimensional echocardiography, 24-hour Holter ECG monitoring with HRV and HRT evaluation, and CPET.

\section{Echocardiography}

Two-dimensional echocardiography was performed using standard parasternal, apical, and subcostal views (VIVID 4 GE Medical System with a 2.5 MHz transducer). Left ventricular ejection fraction was calculated from conventional apical two-chamber and four-chamber images using the biplane Simpson technique.

\section{4-hour Holter ECG monitoring and indices of autonomic nervous system activity analysis}

The HRV and HRT were measured using a 24hour Holter ECG, recorded with a 3-channel Spacelabs Lifecard system (Spacelabs, Del Mar Reynolds, Hertford, UK). The HRV indices were calculated after prospective interactive ECG analysis, using the Impresario Space software (Del Mar Reynolds). The measurements of HRV included time domain - standard deviation of all normal RR intervals (SDNN) - and frequency domain indices.

The frequency domain indices were calculated after fast Fourier transformation of five 10-minute ECG segments, recorded between 2 a.m. and 6 a.m. The highest and lowest values were excluded, and the three remaining values were averaged. The power spectrum density of low frequency (LF) $0.04-0.15 \mathrm{~Hz}$, high frequency (HF) $0.15-0.4 \mathrm{~Hz}$, and the low frequency to high frequency ratio (LF/HF) was calculated using standard methodology [9]. 
The measurements of HRT included turbulence onset (TO) and turbulence slope (TS) according to the algorithm described by Schmidt et al. [12]. Parameters of HRT were considered normal when TO $<0 \%$, and TS > $2.5 \mathrm{~ms} / \mathrm{RR}$ interval. The influence of Nordic walking training on ANS activity was assessed by changes (delta) in SDNN, LF, HF, LF/HF ratio, TO and TS resulting from the comparison of these parameters from the beginning and the end of the programme.

\section{Cardiopulmonary exercise treadmill test}

The symptom limited CPET according to a ramp protocol [13] and ESC guidelines [14] was performed using a Schiller treadmill (Carrollton, USA) which was connected to a computerized breathby-breath spiroergometry system (ZAN 600, ZAN Messgeräte $\mathrm{GmbH}$, Germany). Oxygen consumption was measured continuously using breath-bybreath analysis and used as an index of exercise capacity. Peak $\mathrm{VO}_{2}$ was defined as the highest oxygen uptake level achieved during the final $30 \mathrm{~s}$ of CPET.

\section{Exercise training}

Exercise training was planned in line with the current guidelines for CHF patients [11]. The chosen workload reflected individual effort tolerance with regard to: perceived exertion according to the Borg scale [15] and the training heart rate $(H R)$ range established individually for each patient. The training HR was calculated using the method known as HR reserve. This method uses a percentage of the difference between the maximum HR and the resting HR rate, and adds this value to the resting HR. The target training $H R$ was $40-70 \%$ of the $H R$ reserve. Following the baseline evaluation, patients underwent some (3-6) monitored educational exercise training sessions. Subsequently, patients underwent an 8-week home-based telerehabilitation programme. The training session consisted of three parts: a warm-up lasting 5-10 min (breathing and light resistance exercises, calisthenics), a 15-45 min Nordic walking training, and a 5-minute cool-down. The initial duration of the aerobic Nordic walking training depended on the functional capacity in baseline CPET. If baseline peak $\mathrm{VO}_{2}$ was $<14 \mathrm{ml} / \mathrm{kg} / \mathrm{min}$, we started at $10 \mathrm{~min}$ of Nordic walking/day, if baseline peak $\mathrm{VO}_{2}$ was $14-20 \mathrm{ml} / \mathrm{kg} / \mathrm{min}$, we started at $15 \mathrm{~min}$ of Nordic walking/day; and if baseline peak $\mathrm{VO}_{2}$ was $>20 \mathrm{ml} / \mathrm{kg} / \mathrm{min}$, we started at 20 min of Nordic walking/day. Later on we tended to gradually prolong the time allotted to training so that the final period of time would reach 45-60 min. In order to achieve this optimal pro- longed training duration usually 2 weeks needed to elapse. Patients trained five times a week.

The exercise training effectiveness was assessed by changes (delta) in peak $\mathrm{VO}_{2}$ in CPET, resulting from the comparison of these parameters from the beginning and the end of the programme. The methodology of the telerehabilitation model was described previously [16-18].

In brief, all patients in the TG received a special remote device for ECG and telesupervised exercise training - the telerehabilitation set (Pro Plus Company, Poland), which consists of: an EHO mini device and blood pressure measuring and weighing machine. The EHO mini device enabled the ECG data from three pre-cordial leads to be recorded and transmitted via a mobile phone to the monitoring centre.

\section{Usual care}

Patients in CG receiving "usual care" did not receive a formal exercise training prescription and did not undergo supervised rehabilitation. All patients, independent from the treatment group, were given recommendations for appropriate lifestyle changes and self-management according to ESC guidelines [1].

\section{Statistical analysis}

Statistical analyses were performed using SAS software (version 9.2; Cary, NC, USA). The normality of distributions was verified using the Shapiro-Wilk test. To compare normally distributed continuous variables, paired and unpaired $t$-tests were used. To compare changes of variables in time between training and control groups the general linear model with repeated measures adjusted for age and NYHA class was used. The signed-rank test (for changes over time) and the Wilcoxon test (to compare groups) were used for continuous variables that were not distributed normally. Correlations between peak $\mathrm{VO}_{2}$ and $\mathrm{LF} / \mathrm{HF}$ ratio changes were tested with the Spearman-Pearson test. The $\chi^{2}$ or Fisher exact test was used for categorical variables. Values of $p<0.05$ were considered significant.

\section{Results}

Of the 111 patients randomized, 77 were assigned to the TG and 34 to the CG. Thirty-six patients in the TG and 15 patients in the CG were eligible for HRV analysis. The groups were comparable in terms of demographic data, baseline clinical parameters and pharmacotherapy except for age and NYHA class. It needs to be stressed that all patients were treated with a $\beta$-blocker. The baseline clinical characteristics of patients are shown in Table I. 
Table I. Baseline characteristics

\begin{tabular}{|c|c|c|c|}
\hline Characteristics & $\begin{array}{l}\text { Training group } \\
\quad(n=36)\end{array}$ & $\begin{array}{l}\text { Control group } \\
\quad(n=15)\end{array}$ & Value of $p$ \\
\hline Males & $31(86)$ & $15(100)$ & NS \\
\hline Age [years] & $52.6 \pm 10.12$ & $61.4 \pm 13.2$ & 0.0205 \\
\hline Left ventricular ejection fraction (\%) & $32 \pm 7$ & $33 \pm 8$ & NS \\
\hline \multicolumn{4}{|l|}{ Etiology of heart failure: } \\
\hline Ischaemic & $25(69.4)$ & $12(80.0)$ & NS \\
\hline Non-ischaemic & $11(30.6)$ & $3(20.0)$ & NS \\
\hline \multicolumn{4}{|l|}{ Past medical history: } \\
\hline Myocardial infarction & $24(66.7)$ & $12(80.0)$ & NS \\
\hline Angioplasty & $18(50.0)$ & $10(66.7)$ & NS \\
\hline Coronary artery bypass grafting & $6(11.8)$ & $4(26.7)$ & NS \\
\hline Stroke & $1(2.9)$ & $1(6.7)$ & NS \\
\hline Diabetes & $8(22.2)$ & $4(26.67)$ & NS \\
\hline Hyperlipidaemia & $19(52.8)$ & $8(53.3)$ & NS \\
\hline \multicolumn{4}{|l|}{ Functional status: } \\
\hline NYHA II & $32(88.9)$ & $8(53.3)$ & 0.009 \\
\hline NYHA III & $4(11.1)$ & $7(46.7)$ & 0.009 \\
\hline \multicolumn{4}{|l|}{ Heart rate variability: } \\
\hline SDNN [ms] & $118 \pm 30$ & $124 \pm 32$ & NS \\
\hline Low frequency $\left[\mathrm{ms}^{2} / \mathrm{Hz}\right]$ & $532 \pm 601$ & $339 \pm 211$ & NS \\
\hline High frequency $\left[\mathrm{ms}^{2} / \mathrm{Hz}\right]$ & $392 \pm 493$ & $225 \pm 154$ & NS \\
\hline Low-to-high frequency ratio & $1.91 \pm 1.11$ & $2.2 \pm 1.6$ & NS \\
\hline \multicolumn{4}{|l|}{ Heart rate turbulence: } \\
\hline Turbulence onset (\%) & $-1.51 \pm 3.12$ & $-1.31 \pm 1.13$ & NS \\
\hline Turbulence slope [ms/RR interval] & $9.04 \pm 10.29$ & $5.43 \pm 5.01$ & NS \\
\hline \multicolumn{4}{|l|}{ Cardiopulmonary exercise test: } \\
\hline Peak $\mathrm{VO}_{2}[\mathrm{ml} / \mathrm{kg} / \mathrm{min}]$ & $16.98 \pm 4.02$ & $17.90 \pm 3.61$ & NS \\
\hline \multicolumn{4}{|l|}{ Treatment: } \\
\hline$\beta$-Blocker & $36(100)$ & $15(100)$ & NS \\
\hline Angiotensin converting enzyme inhibitors & $32(88.9)$ & $13(86.7)$ & NS \\
\hline Angiotensin receptor blockers & $4(11.1)$ & $1(6.7)$ & NS \\
\hline Loop diuretics & $19(52.8)$ & $8(53.3)$ & NS \\
\hline Aspirin & $29(80.6)$ & $11(73.3)$ & NS \\
\hline Anticoagulants & $8(22.2)$ & $4(26.7)$ & NS \\
\hline Statins & $28(77.8)$ & $11(73.3)$ & NS \\
\hline Implantable cardioverter-defibrillator & $24(66.7)$ & $9(60.0)$ & NS \\
\hline
\end{tabular}

Data presented are mean \pm SD or $n$ (\%), NS - non-significant, NYHA - New York Heart Association class, SDNN - standard deviation of all normal RR intervals, peak $\mathrm{VO}_{2}-$ peak oxygen consumption. 
Table II. Comparison of outcomes before and after telerehabilitation based on Nordic walking training in the training group $\left(p^{1}\right)$ and before and after observation in the control group $\left(p^{2}\right)$ and between groups - group $\times$ time adjusted for age and New York Heart Association class $\left(p^{3}\right)$

\begin{tabular}{|c|c|c|c|c|c|c|c|}
\hline \multirow[t]{2}{*}{ Parameter } & \multicolumn{3}{|c|}{ Training group } & \multicolumn{3}{|c|}{ Control group } & \multirow{2}{*}{$\begin{array}{l}\text { Value } \\
\text { of } p^{3}\end{array}$} \\
\hline & $\begin{array}{l}\text { Before } \\
\text { training }\end{array}$ & $\begin{array}{c}\text { After } \\
\text { training }\end{array}$ & $\begin{array}{l}\text { Value } \\
\text { of } p^{1}\end{array}$ & $\begin{array}{c}\text { Before } \\
\text { observation }\end{array}$ & $\begin{array}{c}\text { After } \\
\text { observation }\end{array}$ & $\begin{array}{l}\text { Value } \\
\text { of } p^{2}\end{array}$ & \\
\hline SDNN [ms] & $118 \pm 30$ & $119.5 \pm 31$ & NS & $124 \pm 32$ & $123 \pm 25$ & NS & NS \\
\hline $\mathrm{LF}\left[\mathrm{ms}^{2} / \mathrm{Hz}\right]$ & $532 \pm 601$ & $440 \pm 572$ & NS & $339 \pm 211$ & $699 \pm 1112$ & NS & NS \\
\hline $\mathrm{HF}\left[\mathrm{ms}^{2} / \mathrm{Hz}\right]$ & $392 \pm 493$ & $482 \pm 535$ & NS & $225 \pm 154$ & $386 \pm 540$ & NS & NS \\
\hline LF/HF & $1.91 \pm 1.11$ & $1.07 \pm 0.63$ & 0.0001 & $2.2 \pm 1.6$ & $2.46 \pm 1.6$ & NS & 0.0038 \\
\hline TO (\%) & $-1.51 \pm 3.12$ & $-2.14 \pm 2.69$ & NS & $-1.31 \pm 1.13$ & $-1.99 \pm 1.50$ & NS & NS \\
\hline $\begin{array}{l}\text { TS [ms/RR } \\
\text { interval] }\end{array}$ & $9.04 \pm 10.29$ & $8.88 \pm 7.16$ & NS & $5.43 \pm 5.01$ & $5.98 \pm 3.23$ & NS & NS \\
\hline
\end{tabular}

Data presented are mean values \pm SD; statistical significance: $p<0.05 ; N S-$ non-significant, SDNN - standard deviation of all normal RR intervals; LF - low frequency, HF - high frequency; low-to-high frequency ratio; TO-turbulence onset, TS - turbulence slope.

\section{Effects of Nordic walking training on autonomic nervous system indices}

There was a significant improvement in sympathovagal balance evaluated by LF/HF ratio only in the TG $(p=0.0001)$, which was statistically significant in between-group analysis after adjustment for age and NYHA class ( $p=0.0038)$. The HRT did not change significantly in either group. The details are presented in Table II.

\section{Effectiveness of Nordic walking training}

Nordic walking training resulted in a significant improvement in functional capacity assessed by peak $\mathrm{VO}_{2}(16.98 \pm 4.02$ vs. $19.70 \pm 4.36 \mathrm{ml} / \mathrm{kg} /$ min, $p<0.0001)$. In the CG we did not observe significant changes in peak $\mathrm{VO}_{2}(17.90 \pm 3.61$ vs. $17.72 \pm 3.95, p=0.7805)$. Between-group analysis showed that the differences between TG and CG were statistically significant in terms of $\Delta$ peak $\mathrm{VO}_{2}$, after adjustment for age and NYHA class $(p=0.0081)$ (Figure 1).
Additionally, an inverse correlation was found between the decrease in $\Delta \mathrm{LF} / \mathrm{HF}$ and the increase in $\Delta$ peak $\mathrm{VO}_{2}(R=-0.3830, p=0.0211)$ only in the TG (Figure 2). There was no correlation observed between these parameters in the CG $(R=-0.2163$, $p=0.4387$ ) (Figure 3).

\section{Discussion}

The main and novel finding of our study is that in CHF patients, home-based telemonitored Nordic walking training leads to the improvement of the balance of the ANS components assessed by LF/HF ratio and physical capacity evaluated by peak $\mathrm{VO}_{2}$ in CPET. Moreover, the changes in peak $\mathrm{VO}_{2}$ were significantly correlated with the changes in LF/HF ratio. In the TG, individual ANS parameters (SDNN, LF, HF) indicated positive yet non-significant statistical trends (decreased LF, increased $\mathrm{HF}$ ), but in consequence, final balance of the ANS components (LF/HF) improved significantly.

For this reason, it is clear that the positive yet statistically non-significant changes of individual

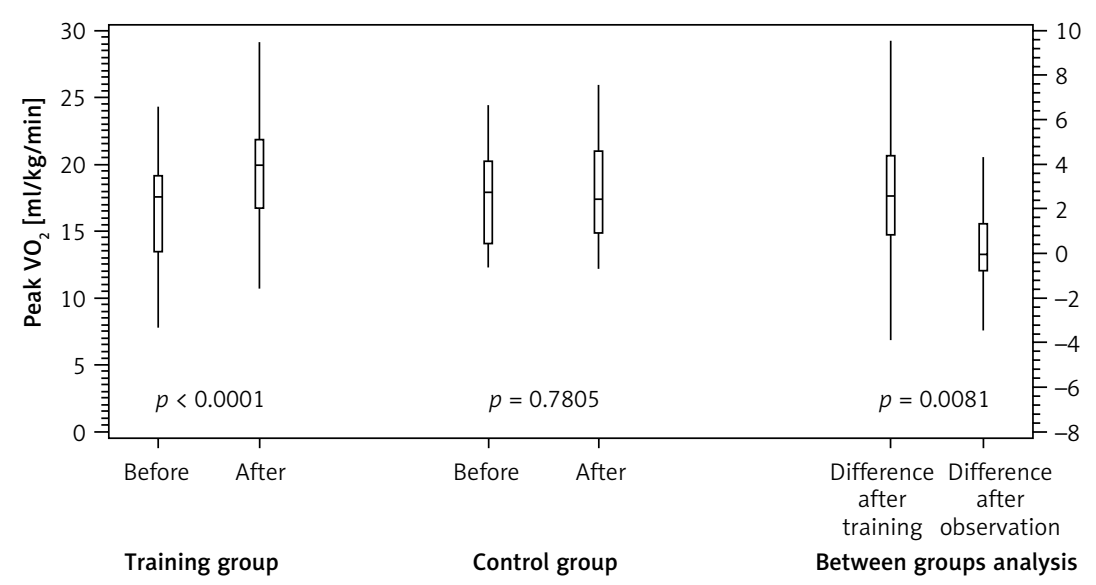

Figure 1. Changes in peak oxygen consumption (peak $\mathrm{VO}_{2}$ ) within training and control groups and between those groups 


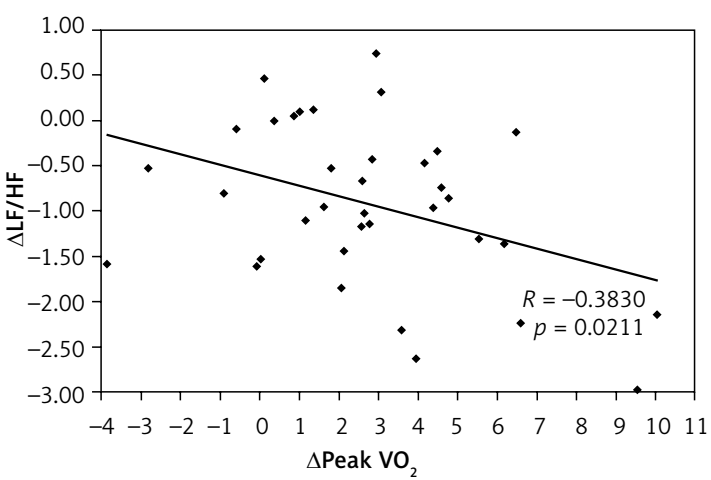

Figure 2. Correlation between the change in peak oxygen consumption ( $\Delta$ peak $\left.\mathrm{VO}_{2}\right)$ and the change in low-to-high frequency ratio $(\Delta \mathrm{LF} / \mathrm{HF})$ in training group

ANS parameters which caused a significant influence on the parasympathetic-sympathetic balance can be considered as one of the pleiotropic (i.e. multifactorial, broader) effects of Nordic walking training. These favourable changes were not observed in the CG. The HRT did not change significantly in either the training or the control group. It needs to be stressed that before and after the 8-week Nordic walking training/observation the TO and TS values were within the norm in both groups. The varied response to Nordic walking in terms of HRV and HRT can be explained by the fact that ANS indices analyzed in this study reflect different components of the ANS. While HRV reflects its spontaneous activity, HRT reflects the response of baroreflex receptor activity provoked by ventricular premature beats. So far, only one study focused on HRT after rehabilitation in CHF patients has been published and our observations are concordant with this specific study [19].

It is clear that the ANS reaction to exercise training depends on the ANS dysfunction level prior to rehabilitation. For the results of our study it is essential that both groups were comparable in terms of analyzed parameters responsible for ANS activity precisely prior to the intervention and, for this reason, the improvement of parasympathetic-sympathetic balance after the rehabilitation in the TG as compared to the CG can be considered reliable and valid.

Few studies of CHF patients showed favourable effects on HRV after cardiac rehabilitation. Although published studies have evaluated the influence of exercise training on ANS, the influence of Nordic walking training on HRV and HRT has not yet been assessed in any study. The majority of published data indicates that endurance aerobic training significantly increases SDNN [20-23] Nevertheless, Selig et al. did not find any difference after moderate-intensity resistance exercise training in the time domain (SDNN) but observed significant improvement in LF/HF ratio [24]. Our

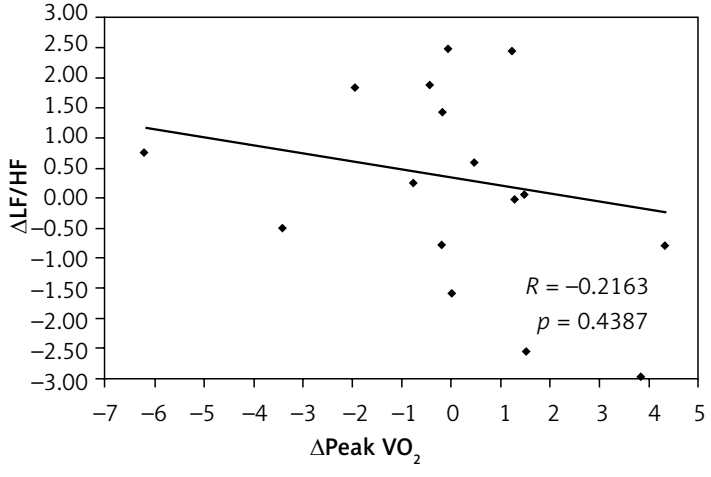

Figure 3. Correlation between the change in peak oxygen consumption ( $\Delta$ peak $\mathrm{VO}_{2}$ ) and the change in low-to-high frequency ratio $(\Delta \mathrm{LF} / \mathrm{HF})$ in control group

results are consistent with these outcomes. Coats et al., on the other hand, reported that exercise training improved exercise tolerance, increased HRV and reduced whole body norepinephrine spillover in CHF patients [21]. Kiilavuori et al. showed that rehabilitation increased high frequency HRV in patients suffering from CHF [25]. Their findings support the hypothesis that exercise training modulates autonomic derangement in CHF by increasing parasympathetic tone. Radaelli et al. found that sympathetic modulation of peripheral vessels is diminished in CHF but significantly improved following the rehabilitation [26]. The training appears to impart beneficial changes in autonomic control in CHF patients through both increased sympathetic control of peripheral vessels and parasympathetic control of heart rate [26].

Malfato et al. observed greater restoration of ANS responsiveness to vagal and sympathetic stimulation in CHF patients after 3 months of a low-intensity rehabilitation programme. Moreover, the authors reported significant improvement not only in ANS activity but also in peak $\mathrm{VO}_{2}$ [27]. In contrast to our study there was no correlation between the amount of improvement in exercise capability (expressed as \% change in peak $\mathrm{VO}_{2}$ ) and the amount of change in autonomic responsiveness (expressed as changes in LF/HF during controlled respiration or during standing) [27]. Also Murad et al. reported that the improvement in HRV did not correlate with measures of exercise capacity [28]. Similarly, our study proved that there were no positive effects in patients who did not undergo the exercise training.

The studies conducted so far evaluated various parameters (time domain and/or frequency domain indices) reflecting ANS activity. Individual parameters have not always been improved, yet the sympathetic-parasympathetic balance was restored, which indicated that exercise training increases vagal tone and reduces sympathetic cardiac influence, resulting in the improvement in 
HRV. On the whole, all the studied exercise modalities such as supervised aerobic endurance and resistance training and home-based rehabilitation improved HRV among CHF patients [19-32].

The findings to date imply that exercise training exerts an influence on HRV favourable modulation of sympathetic/parasympathetic balance (via increasing vagal modulation and decreasing sympathetic tone). Further research is needed to identify the training protocols which would produce optimal improvements in HRV and HRT. This research should encompass the assessment of the following variables: training type (endurance/ resistance/strength/respiratory), intensity (aerobic/anaerobic), method (continuous/interval), duration (time of session), frequency (number of sessions per day/per week) application (systemic/regional), control (supervised/telesupervised/ unsupervised), progression (consider low level initially/increase if tolerated) and setting (hospital/ out-patient/home-based) [2-4, 11, 33].

For this reason, additional studies comparing the influence of different training modalities on ANS activity on larger patient groups are necessary.

The limitations include the fact that our study was part of a single centre trial. Due to the nature of the intervention, the study was not blinded. The study group was not very large. Only a few women participated in the study; therefore our outcomes cannot be fully extrapolated to the female population.

The duration of Nordic walking training in our study was relatively short - only 8 weeks. We did not exclude patients who underwent $C A B G$, in whom afferent and efferent cardiac innervations might have been impaired. We also did not exclude patients suffering from diabetes mellitus and hypertension in whom ANS might have been affected by these diseases. Nevertheless, both training and control groups were comparable in terms of CABG, diabetes mellitus and hypertension.

In conclusion, Nordic walking showed positive effects as far as the parasympathetic-sympathetic balance in CHF patients of NYHA class II and III was concerned, yet no significant changes in HRT were observed. This positive balance change correlates with the improvement of physical capacity resulting from home-based telemonitored Nordic walking training modality. Our results demonstrate that different processes modulate HRV and HRT, which offers additional information about ANS activity. The 8-week Nordic walking training was nevertheless unsuccessful in terms of completely normalizing the ANS function.

\section{Acknowledgments}

The study was supported by the National Science Centre, Poland - grant number NN404 107936.

\section{Conflict of interest}

The authors declare no conflict of interest.

\section{References}

1. McMurray JJ, Adamopoulos S, Anker SD, et al. ESC guidelines for the diagnosis and treatment of acute and chronic heart failure 2012. Eur Heart J 2012; 33: 1787-869.

2. Kasznicki J, Drzewoski J. Heart failure in the diabetic population - pathophysiology, diagnosis and management. Arch Med Sci 2014; 10: 546-56.

3. Lainscak M, Letonja M, Kovacic D, et al. General public awareness of heart failure: results of questionnaire survey during Heart Failure Awareness Day 2011. Arch Med Sci 2014; 10: 355-60.

4. Anker MS, von Haehling S, Springer J, Banach M, Anker SD. Highlights of mechanistic and therapeutic cachexia and sarcopenia research 2010 to 2012 and their relevance for cardiology. Arch Med Sci 2013; 9: 166-71.

5. Routledge FS, Campbell TS, McFetridge-Durdle JA, et al. Improvements in heart rate variability with exercise therapy. Can J Cardiol 2010; 26: 303-12.

6. Gademan MGJ, Swenne CA, Verwey HF, et al. Effect of exercise training on autonomic derangement and neurohumoral activation in chronic heart failure. J Cardiac Fail 2007; 13: 294-303.

7. Braith RW, Edwards DG. Neurohormonal abnormalities in heart failure: impact of exercise training. Congest Heart Fail 2003; 9: 70-6.

8. Tschentscher M, Niederseer D, Niebauer J. Health benefits of Nordic walking: a systematic review. Am J Prev Med 2013; 44: 76-84.

9. Task Force of The European Society of Cardiology and The North American Society of Pacing and Electrophysiology. Heart rate variability. Standards of measurement, physiological interpretation, and clinical use. Eur Heart J 1996; 17: 354-81.

10. Piotrowicz E, Zielinski T, Bodalski R, et al. Home-based telemonitored Nordic walking training is well accepted, safe, effective and has high adherence among heart failure patients, including those with cardiovascular implantable electronic devices - a randomized controlled study. Eur J Prev Cardiol 2015; 22: 1368-77.

11. Piepoli MF, Conraads V, Corra U, et al. Exercise training in heart failure: from theory to practice. A consensus document of the HFA and the EACPR. Eur J Heart Fail 2011; 13: 347-57.

12. Schmidt G, Malik M, Barthel P, et al. Heart-rate turbulence after ventricular premature beats as a predictor of mortality after acute myocardial infarction. Lancet 1999; 353: 1390-6.

13. Balady GJ. Medical evaluation and exercise testing. In: Guidelins for Cardiac Rehabilitation and Secondary Prevention Programs. Williams MA (ed.). Champaign (IL), Human Kinetics 2004; 79.

14. Piepoli MF, Corrà U, Agostoni PG, et al. Statement on cardiopulmonary exercise testing in chronic heart failure due to left ventricular dysfunction: recommendations for performance and interpretation Part II: How to perform cardiopulmonary exercise testing in chronic heart failure. Eur J Cardiovasc Prev Rehabil 2006; 13: 300-11.

15. Borg GA. Psychophysical bases of perceived exertion. Med Sci Sports Exerc 1982; 14: 377-81.

16. Piotrowicz E. How to do telerehabilitation in heart failure patients. Cardiol J 2012; 19: 243-8. 
17. Piotrowicz E, Baranowski R, Bilinska $M$, et al. A new model of home-based telemonitored cardiac rehabilitation in patients with heart failure: effectiveness, quality of life, and adherence. Eur J Heart Fail 2010; 12: 164-71.

18. Piotrowicz E, Jasionowska A, Banaszak-Bednarczyk $M$, et al. ECG telemonitoring during home-based cardiac rehabilitation in heart failure patients. J Telemed Telecare 2012; 18: 193-7.

19. Piotrowicz E, Baranowski R, Piotrowska M, Zieliński T, Piotrowicz R. Variable effects of physical training of heart rate variability, heart rate recovery, and heart rate turbulence in chronic heart failure. Pacing Clin Electrophysiol 2009; 32 (Suppl. 1): S113-5.

20. Adamopoulos S, Ponikowski P, Cerquetani E, et al. Circadian pattern of heart rate variability in chronic heart failure patients. Effects of physical training. Eur Heart J 1995; 16: 1380-6.

21. Coats AJ, Adamopoulos S, Radaelli A, et al. Controlled trial of physical training in chronic heart failure. Exercise performance, hemodynamics, ventilation, and autonomic function. Circulation 1992; 85: 2119-31.

22. Larsen Al, Gjesdal K, Hall C, et al. Effect of exercise training in patients with heart failure: a pilot study on autonomic balance assessed by heart rate variability. Eur J Cardiovasc Prev Rehabil 2004; 11: 162-7.

23. European Heart Failure Training Group. Experience from controlled trials of physical training in chronic heart failure. Protocol and patient factors in effectiveness in the improvement in exercise tolerance. Eur Heart J 1998; 19: 466-75.

24. Selig SE, Carey MF, Menzies DG, et al. Moderate-intensity resistance exercise training in patients with chronic heart failure improves strength, endurance, heart rate variability, and forearm blood flow. J Card Fail 2004; 10: 21-30.

25. Kiilavuori K, Toivonen L, Naveri H, et al. Reversal of autonomic derangements by physical training in chronic heart failure assessed by heart rate variability. Eur Heart J 1995; 16: 490-5.

26. Radaelli A, Coats AJ, Leuzzi S, et al. Physical training enhances sympathetic and parasympathetic control of heart rate and peripheral vessels in chronic heart failure. Clin Sci (Lond) 1996; 91 Suppl.: 92-4.

27. Malfatto G, Branzi G, Riva B, et al. Recovery of cardiac autonomic responsiveness with low-intensity physical training in patients with chronic heart failure. Eur J Heart Fail 2002; 4: 159-66.

28. Murad K, Brubaker PH, Fitzgerald DM, et al. Exercise training improves heart rate variability in older patients with heart failure: a randomized, controlled, single-blinded trial. Congest Heart Fail 2012; 18: 192-7.

29. Iellamo F, Manzi V, Caminiti G, et al. Dose-response relationship of baroreflex sensitivity and heart rate variability to individually-tailored exercise training in patients with heart failure. Int J Cardiol 2013; 166: 334-9.

30. Pietilä M, Malminiemi K, Vesalainen R, et al. Exercise training in chronic heart failure: beneficial effects on cardiac (11)C-hydroxyephedrine PET, autonomic nervous control, and ventricular repolarization. J Nucl Med 2002; 43: 773-9.

31. Ponikowski P, Chua TP, Piepoli $M$, et al. Ventilatory response to exercise correlates with impaired heart rate variability in patients with chronic congestive heart failure. Am J Cardiol 1998; 82: 338-44.

32. Grant CC, Viljoen M, Janse van Rensburg DCJ, et al. Heart rate variability assessment of the effect of physical training on autonomic cardiac control. Ann Noninvasive Electrocardiol 2012; 17: 219-29.
33. Piotrowicz E, Orzechowski P, Chrapowicka A, Piotrowicz R. How should we advise heart failure patients on exercise and what should we tell them? Curr Heart Fail Rep 2014; 11: 274-80. 